ARTICLE

DOI: $10.1038 / \mathrm{s} 41467-018-03065-7$

\title{
Absence of warmth permits epigenetic memory of winter in Arabidopsis
}

Jo Hepworth (1) 1, Rea L. Antoniou-Kourounioti (1) 1, Rebecca H. Bloomer ${ }^{1}$, Catja Selga (1) 2, Kristina Berggren (D) ${ }^{3}$, Deborah Cox', Barley R. Collier Harris', Judith A. Irwin (1) 1, Svante Holm (1D ${ }^{3}$, Torbjörn Säll', Martin Howard (1) ${ }^{1} \&$ Caroline Dean ${ }^{1}$

Plants integrate widely fluctuating temperatures to monitor seasonal progression. Here, we investigate the temperature signals in field conditions that result in vernalisation, the mechanism by which flowering is aligned with spring. We find that multiple, distinct aspects of the temperature profile contribute to vernalisation. In autumn, transient cold temperatures promote transcriptional shutdown of Arabidopsis FLOWERING LOCUS C (FLC), independently of factors conferring epigenetic memory. As winter continues, expression of VERNALIZATION INSENSITIVE3 (VIN3), a factor needed for epigenetic silencing, is upregulated by at least two independent thermosensory processes. One integrates long-term cold temperatures, while the other requires the absence of daily temperatures above $15^{\circ} \mathrm{C}$. The lack of spikes of high temperature, not just prolonged cold, is thus the major driver for vernalisation. Monitoring of peak daily temperature is an effective mechanism to judge seasonal progression, but is likely to have deleterious consequences for vernalisation as the climate becomes more variable.

\footnotetext{
${ }^{1}$ John Innes Centre, Norwich Research Park, Norwich NR4 7UH, UK. ${ }^{2}$ Department of Biology, Lund University, Sölvegatan 35, Lund 223 62, Sweden. ${ }^{3}$ Faculty of Science, Technology and Media, Department of Natural Sciences, Mid Sweden University, Sundsvall SE-851 70, Sweden. Jo Hepworth and Rea L. Antoniou-Kourounioti contributed equally. Correspondence and requests for materials should be addressed to M.H. (email: martin.howard@jic.ac.uk) or to C.D. (email: caroline.dean@jic.ac.uk)
} 
Sarat easonal cues are central to the timing of many developmental transitions in biology. Plants use prolonged cold exposure to align the transition to flowering with spring, a process called vernalisation. In Arabidopsis, vernalisation involves the epigenetic silencing of FLOWERING LOCUS C (FLC) by the conserved Polycomb Repressive Complex 2 (PRC2) in combination with PHD proteins, including cold-induced VERNALIZATION INSENSITIVE3 $\left(\right.$ VIN3) ${ }^{1-5}$. Vernalisation can occur at constant temperatures between 0 and $15^{\circ} \mathrm{C}^{6-8}$, but plants in the field experience daily fluctuations of more than $10^{\circ} \mathrm{C}$, equivalent in some locations (e.g. Norwich, UK) to the difference in average temperature between autumn and winter. Experiments indicate that plants are able to extract a reliable signal from this noisy and variable temperature profile ${ }^{8-11}$, but how they integrate these signals over timescales of many weeks to judge the passing of winter has not been clear ${ }^{10,12,13}$. Previous analyses have developed cumulative measures of cold exposure over time, including photothermal units or 'degree days', used to plan the harvest dates of crops ${ }^{9,14-17}$. However, the molecular mechanisms underlying these measures are unknown.

Here, we test expression of FLC and VIN3 in the field and in controlled experiments to find that there are two phases of FLC downregulation with distinct temperature sensitivities, one independent and one dependent on VIN3. VIN3 itself is controlled by at least two thermosensors and the circadian clock, which combine to only permit expression when temperatures cease to rise daily above $15^{\circ} \mathrm{C}$. The absence of warmth is therefore a key signal of winter for Arabidopsis.

\section{Results}

FLC silencing can be separated into two phases in the field. To understand the process of vernalisation in natural conditions, we established field experiments (Fig. 1a) in very different winter conditions: a site in Norwich (natural conditions but under glass), and fields in South Sweden and North Sweden, with sowings two weeks apart for the latter to defend against loss to early frost (Supplementary Fig. 1a-c). We monitored the temperature and the expression of FLC and VIN3 to measure the progress of vernalisation in the standard vernalisation-requiring Arabidopsis thaliana genotype Col FRI ${ }^{S F 2}$ (Fig. 1b, c).

Daily ground level temperatures fluctuated widely in Sweden in the early autumn, but the range of fluctuations narrowed in late autumn and early winter as vernalisation occurred, with the temperature at times becoming constant in late winter due to insulating snow cover. In Norwich, the daily temperature variation was also large in the autumn, behaviour which persisted for longer than in Sweden. At all sites FLC transcriptional downregulation was detected within the first 40 days. In both Swedish experiments, the FLC expression was well described by an exponential decay over time (Fig. 1c), with expression in South Sweden showing a faster rate of decay than in the North (slope significantly different as tested by Analysis of Covariance (ANCOVA), $p=5.30 \times 10^{-9}$ ) (Fig. 1d). The plants from the two sowing groups in the North showed very similar FLC profiles (Supplementary Fig. 1a-c). VIN3 showed significant upregulation in the first few weeks of sampling in all Swedish sites, albeit more slowly in the first North experiment than the South (Fig. 1c, Supplementary Figs. $1 \mathrm{~b}$ and $2 \mathrm{a}, \mathrm{b}$ ). In Norwich, however, the initial downregulation of FLC was slow, but later became more rapid than in Sweden $\left(p=1.01 \times 10^{-9}\right.$ for slope comparison between Norwich after 55 days and South Sweden) (Fig. 1d), with VIN3 expression not significantly upregulated until nearly two months after sowing (Fig. 1c). The data for unspliced FLC are more variable (Supplementary Fig. 2c), but the faster rate of shutdown in Norwich compared to Sweden is still apparent, suggesting a reduction in transcription rather than an increase in mRNA degradation rate. It is known that vernalisation efficiency decreases at temperatures close to $0{ }^{\circ} \mathrm{C}$, which may explain the slower rate during the very low temperature period in Sweden ${ }^{6,8,9}$.

We hypothesised that the changed VIN3 behaviour in Norwich, as compared to both Swedish sites, could be the cause of the differences in the FLC transcriptional shutdown. We found that FLC downregulation in Norwich could be split into two exponential decay regimes with different characteristic rates: the first with a shallower gradient before VIN3 upregulation, the second with a steeper gradient during VIN3 upregulation (Fig. 1e, Supplementary Fig. 1d). In a vin3-4 FRI null mutant, and mutants of the PRC2 protein VERNALIZATION 2 (VRN2) $)^{2,4,5}$, the second, faster phase was absent and the slower first phase continued for the whole winter, supporting a causative role for VIN3 induction and PRC2 in the faster rate (Fig. 1e, Supplementary Fig. 1e). This reduced rate of silencing led to higher FLC levels in these mutants, resulting in later flowering at the end of the winter (Supplementary Fig. 3a).

These experiments demonstrated that the VIN3-independent and the VIN3-PRC2-dependent aspects of vernalisation are, in principle, temperature separable phases. Initial transcriptional shutdown is thought to be a requirement for heritable, PRC2driven epigenetic silencing ${ }^{18-20}$. However, at FLC, in constantcondition laboratory experiments and the Swedish field, VIN3 upregulation occurs simultaneously with VIN3-independent transcriptional shutdown, so the two phases are superimposed (except in PHD-PRC2 mutants) ${ }^{21,22}$. In Norwich, the temperature profile temporally separates these phases, indicating that they are driven by different thermosensory mechanisms.

FLC responds to temperature extremes. To understand the temperature dependence of both the PRC2-independent and -dependent phases, we investigated the effects of four distinct controlled temperature profiles on VIN3 and FLC. Two of the profiles maintained constant temperatures $\left(8^{\circ} \mathrm{C}\right.$ and $\left.14.2^{\circ} \mathrm{C}\right)$, with vernalisation occurring at different rates $^{7,8}$. The other two were fluctuating temperature regimes mimicking profiles from the field experiments, with average temperatures matching those of the constant temperature treatments (Fig. 2a).

The rates of increase of VIN3 expression in Col FRI ${ }^{S F 2}$ were very similar in both the constant and fluctuating $8^{\circ} \mathrm{C}$ conditions (Fig. 2b). A similar conclusion held for the rates of decrease of FLC expression (Fig. 2c). These results support the hypothesis that the two vernalisation phases are similarly responsive to temperatures between 5.5 and $11.5^{\circ} \mathrm{C}$. Indeed, in the vin3-4 FRI mutant (Fig. 2d), FLC downregulation was again very similar in response to both treatments, suggesting that the VIN3-independent phase does not distinguish between these temperature profiles.

As previously reported ${ }^{7,8}$, at constant $14.2^{\circ} \mathrm{C}$ as compared to constant $8^{\circ} \mathrm{C}$, both VIN3 and FLC took longer to reach the same level of activation or repression, respectively (Fig. 2b,c). VIN3 was also upregulated more slowly in response to the 14-fluctuating conditions than the 14-constant (Fig. 2b). The average temperature in the two treatments was the same, so the average temperature is not therefore the signal setting VIN3 expression. Furthermore, in the 14-fluctuating profile, temperatures as low as $8{ }^{\circ} \mathrm{C}$ were experienced. These lower temperatures, when given as a constant treatment, induced VIN3 more than a constant $14.2^{\circ} \mathrm{C}$ (Fig. $2 \mathrm{~b}$ and ref. $^{7}$ ). However, the overall effect of a 14-fluctuating profile versus a 14-constant gave much lower VIN3 expression, suggesting that the high temperature spikes (of up to $22^{\circ} \mathrm{C}$ experienced during the 14 -fluctuating cycle) are able to prevent 
a

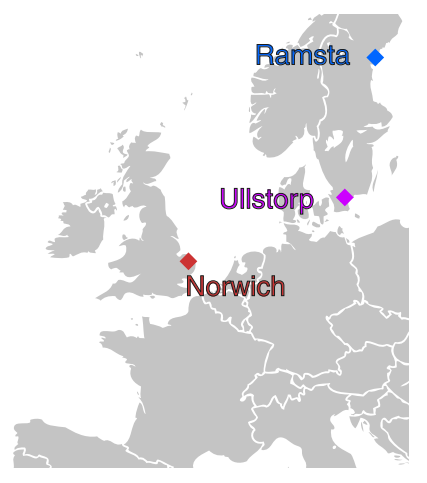

b

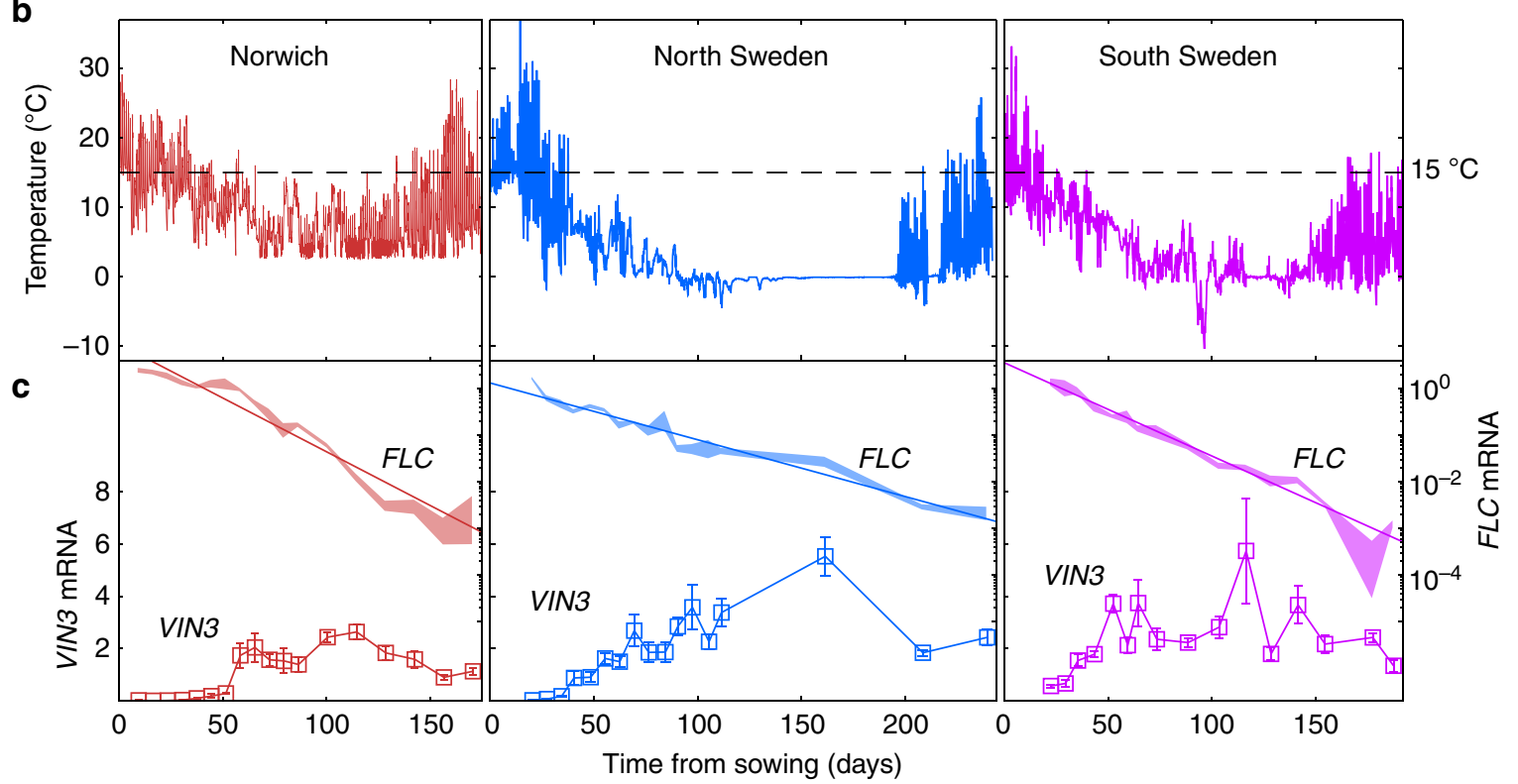

d

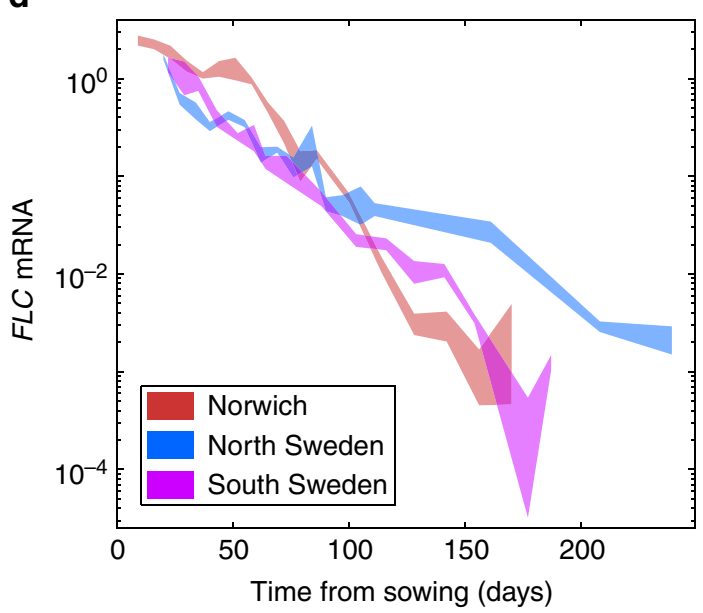

e

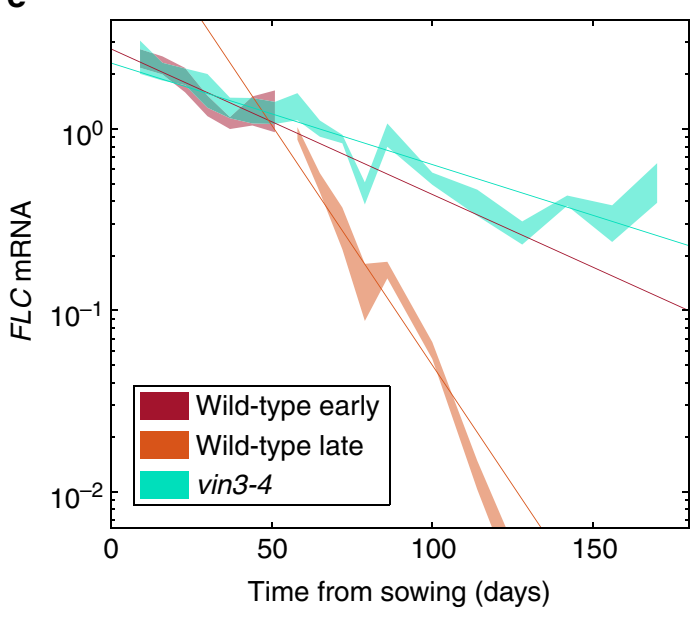

Fig. 1 FLC downregulation occurs in the field in both VIN3-independent and dependent manners. a Locations of field sites in Norwich, North Sweden (Ramsta) and South Sweden (Ullstorp). b Temperatures experienced by the plants in the different sites. Dashed line indicates $15^{\circ} \mathrm{C}$. In Norwich, glasshouse confinement is probably responsible for buffering low temperatures (see Methods). c FLC and VIN3 expression for Col FR/SF2, as measured in the field corresponding to plot above. FLC (top): Thick lines show measured FLC mRNA where c-e thickness of the line represents s.e.m. VIN3 (below): measured expression of VIN3 mRNA, errors = s.e.m. d Comparison of FLC downregulation between different sites. e FLC downregulation in Norwich for 'wild-type' (Col FRI $I^{S F 2}$ ) and a mutant impaired in VIN3 expression (vin3-4 FRI). For the wild-type, the data are separated according to presence (late timepoints) or absence (early timepoints) of VIN3 expression, as measured in panel c. RNA levels in c-e normalised to UBC, PP2A, and internal control. Straight lines (c, e) show the best fit exponential decay profile, fitted by least-squares. $n=2-6$, average $\geq 5$. Map adapted from https://commons.wikimedia.org/wiki/File: Blank_map_of_Europe.svg by maix, under CC BY-SA 2.5 license 

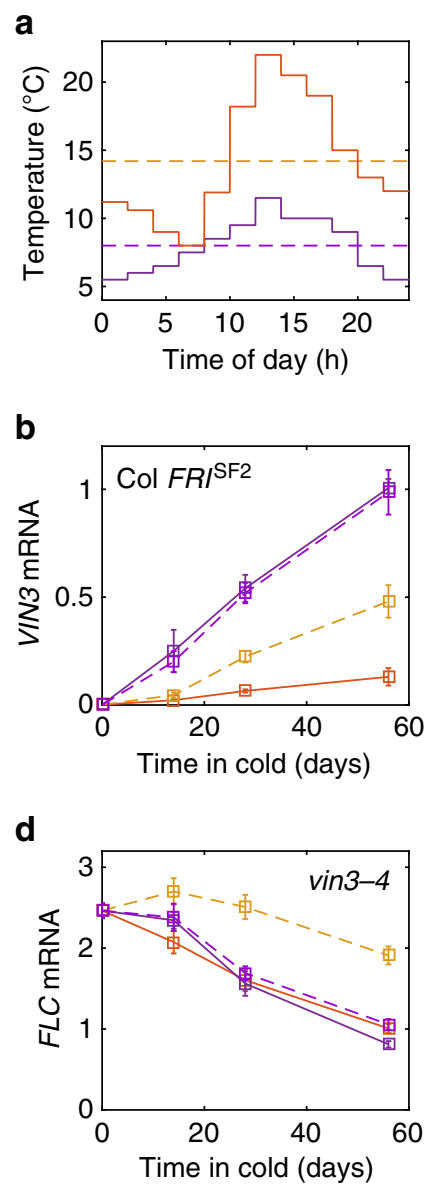

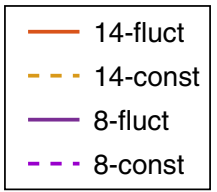

C

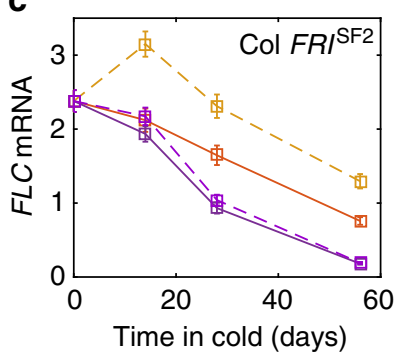

e

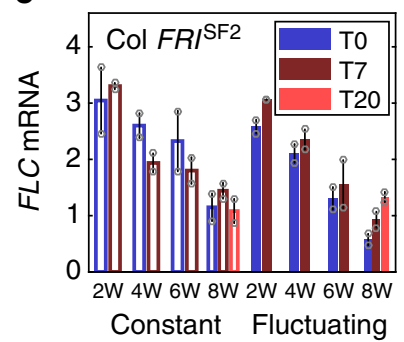

Fig. 2 FLC and VIN3 expression respond to temperature extremes rather than averages. a Temperature profiles of treatments used in the experiment. b, c VIN3 and FLC mRNA from Col FRISF2 plants treated with 'cold' temperature conditions of panel a. For VIN3, plants were sampled 5-7 h after dawn. b $n=3-6$, average $>5.3$. c $n=16-21$, average $>19$. d FLC mRNA in the mutant vin3-4, otherwise as for c. $n=9-21$, average $>17$. e FLC mRNA in Col FRI ${ }^{S F 2}$ during (TO) and after removal (T7, T20) from the $14.2^{\circ} \mathrm{C}$ constant (14-const) or fluctuating (14-fluct) conditions shown in $\mathbf{a}$, $\mathrm{T} x=\mathrm{x}$ days after return to warm, $\mathrm{W}=$ weeks in cold. $n=2$ shown as grey circles. Error bars = s.e.m. In $\mathbf{b}-\mathbf{e}$ RNA levels were normalised to UBC, PP2A

high levels of VIN3 expression. The absence of these warm temperatures thus allows VIN3 to be expressed at higher levels. This phenomenon could also be seen in the Norwich and North Sweden field experiments (Fig. 1b, c, Supplementary Fig. 2a, b), where VIN3 expression was low until daily temperature maxima were regularly lower than $\sim 15^{\circ} \mathrm{C}$.

Surprisingly, FLC expression reached lower levels more rapidly in the 14-fluctuating versus 14-constant conditions (Fig. 2c), despite the former having lower VIN3 expression. This result suggested that the greater degree of downregulation of FLC in the 14-fluctuating conditions is due to the VIN3-independent shutdown. The vin3-4 FRI mutant also showed that VIN3independent downregulation is as effective in 14-fluctuating as it is at either constant or fluctuating $8^{\circ} \mathrm{C}$, whereas 14 -constant is much less effective (Fig. 2d). The simplest interpretation of these results is that the presence of colder temperatures increases the effectiveness of VIN3-independent downregulation and that the lower temperatures in the 14-fluctuating experiment are sufficient to trigger this response. This conclusion may explain results reported by Burghardt et al. in which high-FLC genotypes flowered more rapidly when kept in similar fluctuating

a

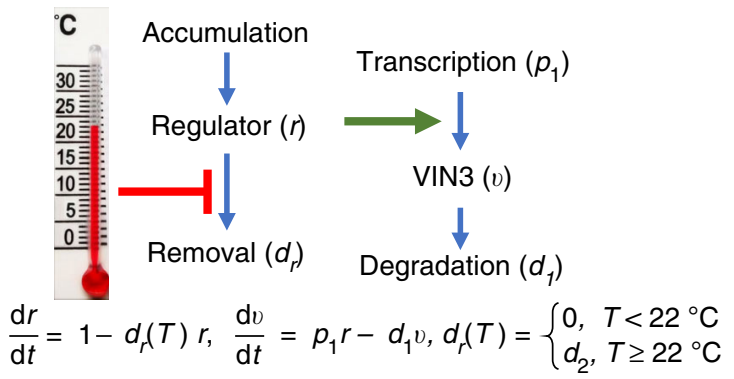

b

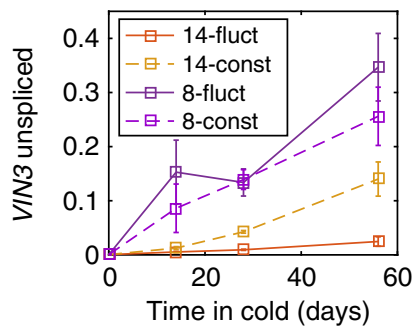

d

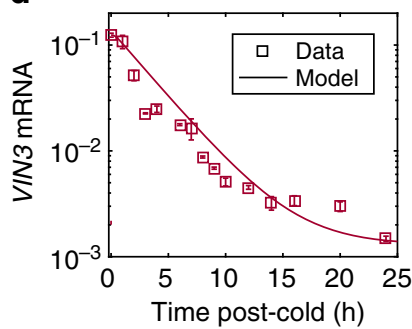

C

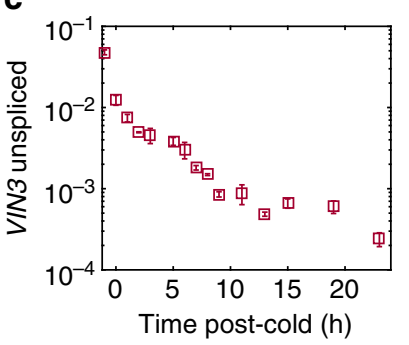

e

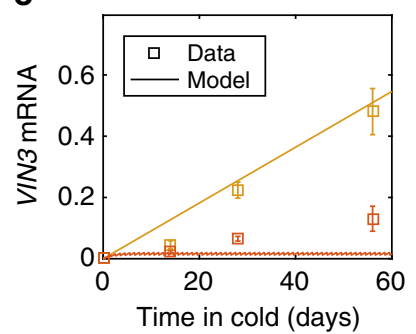

Fig. 3 Modelling VIN3 dynamics reveals its temperature inputs. a Minimal model of VIN3 temperature sensing assuming a single thermosensor $\boldsymbol{r}$ whose levels quantitatively regulate VIN3 transcription. $p_{1}=0.91 \mathrm{day}^{-1}, d_{1}=$ 100 day $^{-1}, d_{2}=7$ day $^{-1}$. See Methods for more details. b Slow VIN3 upregulation in the cold for unspliced RNA, sampled 5-7 h after dawn. $n=$ 3-6, average $>4.7$. c, d VIN3 unspliced and spliced RNA levels upon return to warm $\left(22^{\circ} \mathrm{C}\right)$ following 4 weeks at $5^{\circ} \mathrm{C}$. At time 0 , plants were sampled in the cold and subsequently transferred to warm. $n=2-3$, average $>2.6$. e Slow VIN3 mRNA upregulation in the cold, sampled 5-7 h after dawn. Colours correspond to vernalisation treatment as shown in legend to $\mathbf{b} . n=$ 3-6, average $=5$. Model results shown in $\mathbf{d}, \mathbf{e}$. In $\mathbf{b}-\mathbf{e}$, RNA levels were normalised to $U B C, P P 2 A$; error bars $=$ s.e.m.

conditions $^{23}$. Indeed, in the vin3-4 mutant, 14-fluctuating conditions can lower FLC expression sufficiently to allow more rapid flowering than after 14-constant (Supplementary Fig. 3b).

When plants are moved to warm conditions after weeks of cold temperatures, FLC remains silenced ${ }^{24}$. We observed this response in plants treated at 14-constant. However, for plants treated with 14-fluctuating conditions, FLC levels reactivated ( $p=0.0064$ in a one-tailed paired $t$-test), indicating that at least part of the VIN3-independent pathway is not epigenetically stable (Fig. 2e). That reactivation is not always complete is potentially due to nonvernalisation silencing that occurs slowly in the absence of transcription at $F L C^{18}$.

VIN3 is controlled by more than one thermosensor. To understand these results and their implications for temperature sensing in vernalisation, we constructed a simple mathematical model of VIN3 dynamics to allow us to test and potentially reject hypotheses (Fig. 3a and Methods section). We reasoned that long-term temperature sensing is most likely to act on VIN3 
transcription initiation because we observed that both spliced (Fig. 2b) and unspliced (Fig. 3b) VIN3 levels increase slowly over long time periods in the cold. We therefore assumed that VIN3 transcription is regulated by upstream activating thermosensory processes, which we model with the variable $\boldsymbol{r}$ (Fig. 3a). $\boldsymbol{r}$ slowly responds to time in vernalising temperatures, holds the memory of cold during cold exposure and generates the well-established slow rate of VIN3 induction ${ }^{4,25-27}$. This model makes minimal assumptions as to the molecular properties of $\boldsymbol{r}$, so our conclusions are applicable to many underlying mechanisms, including cis-based chromatin control, potentially at the VIN3 locus itself $^{27,28}$.

Focusing on the behaviour of VIN3 around $14.2^{\circ} \mathrm{C}$ (fluctuating and constant) as compared to $22^{\circ} \mathrm{C}$, we next asked whether thermosensory behaviour could be entirely isolated within $r$ or whether other thermosensory inputs are required. Two key features must be captured by the model: the slow increase of VIN3 expression around $14.2^{\circ} \mathrm{C}$ (albeit slower at 14-fluctuating than at 14-constant) (Fig. 2b), and a rapid decrease in the warm at $22^{\circ} \mathrm{C}$ following a cold treatment (Fig. $\left.3 \mathrm{c}, \mathrm{d}\right)^{4,25-27,29}$. The predicted dynamics of $r$ from the model are shown in Supplementary Fig. 4a, b. The degradation of VIN3 must be fast to show the rapid decrease we see in the warm, and therefore VIN3 dynamics closely follow those of $\boldsymbol{r}$ (Supplementary Fig. 4c, d). Incorporating both types of thermosensory regulation (cold accumulation, warm removal) into $\boldsymbol{r}$ generated a model that could successfully capture VIN3 behaviour at 14-constant cold or postcold $22{ }^{\circ} \mathrm{C}$ warm, but not under 14 -fluctuating conditions (Fig. $3 \mathrm{~d}$, e). In this latter case, $\boldsymbol{r}$ is reset every day at high temperatures and therefore VIN3 is never expressed at higher levels, in contradiction to our experiments (Fig. 3e). The model therefore demonstrates that the thermosensory process(es) that lead to the fast decrease in VIN3 in the warm cannot apply to $r$ but must function separately, allowing us to reject a single-thermosensor (r) model for VIN3 regulation.

With this interpretation, there must be at least two thermosensory inputs to VIN3. One, $\boldsymbol{B}$, has the slow characteristics of $\boldsymbol{r}$ in the cold and forms a long-term monitor of cold temperatures, similar to degree days (Methods). This input is coupled with a second, separate input $(\boldsymbol{A})$ that monitors current temperatures and is responsible for the rapid decrease in VIN3 levels at high temperatures, giving the critical absence of warm response for VIN3. Since both spliced and unspliced VIN3 levels decay quickly in the warm (Fig. 3c, d), it is likely that this rapid temperature response is again mediated through control of VIN3 transcription.

VIN3 is regulated by the circadian clock. To further investigate these fast timescale dynamics, we analysed FLC and VIN3 expression during a single day (over $12 \mathrm{~h}$ and also over $24 \mathrm{~h}$, Fig. 4a, b, Supplementary Figs. 5 and 6), using both the constant and fluctuating profiles ( 8 and $14.2^{\circ} \mathrm{C}$ average). FLC mRNA and unspliced transcript levels did not show an expression pattern consistent with a rapid response (within hours) to changes in temperature, as the pattern was similar in both constant and fluctuating conditions (Supplementary Figs. 5b-g and $6 b-i$ ). Furthermore, although FLC expression was variable, we did not observe any clear diurnal pattern. In contrast, for VIN3 mRNA and unspliced levels, we found a strong diurnal pattern in all cases peaking in the afternoon (Figs. 4a, b, Supplementary Figs. 5h-m and $6 \mathrm{j}-\mathrm{q}$ ). As we observed similar patterns for VIN3 in both constant and fluctuating conditions, this pattern cannot be due to a rapid response to changing temperatures (i.e. it is not explained by the $\boldsymbol{A}$ component). The peak of VIN3 expression was higher with longer cold treatments, as we would expect from the a

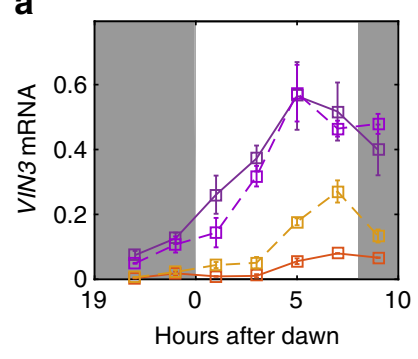

c

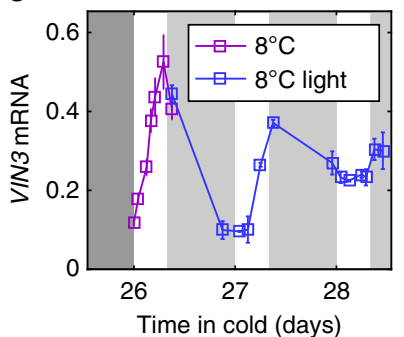

e

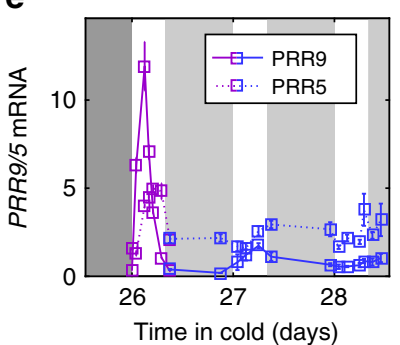

b

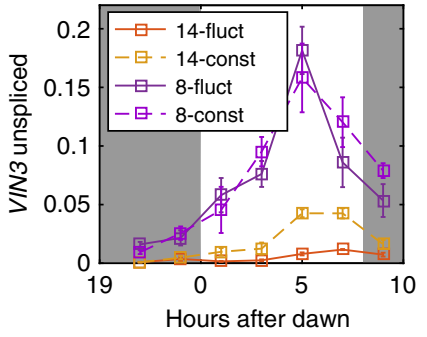

d

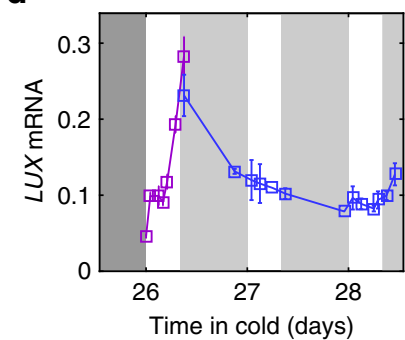

f

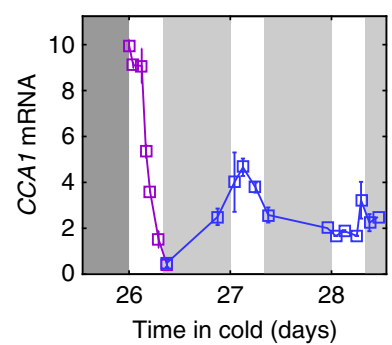

$\mathbf{g}$

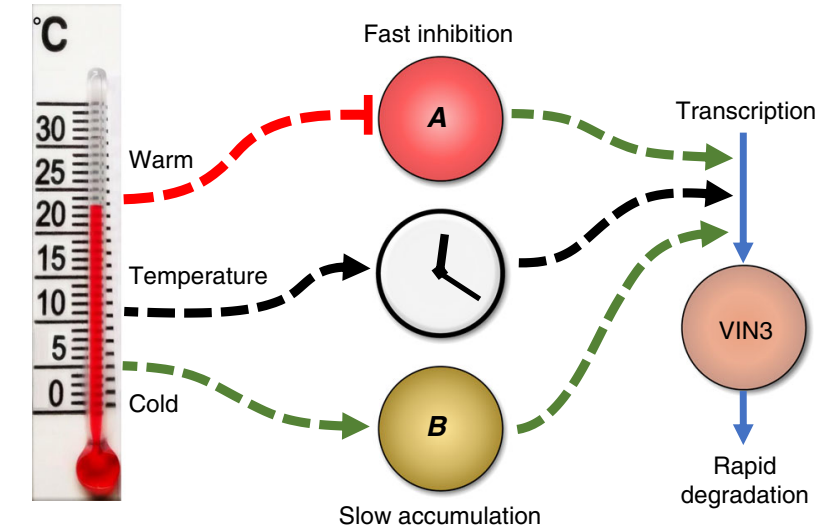

Fig. 4 VIN3 expression is regulated in a circadian manner. a, b VIN3 spliced and unspliced expression over $12 \mathrm{~h}$. Plants grown in $20^{\circ} \mathrm{C}$ night, $22^{\circ} \mathrm{C}$ day $16 \mathrm{~h}$ photoperiod for 1 week and transferred to vernalising $8 \mathrm{~h}$ photoperiod with constant or fluctuating 14.2 or $8^{\circ} \mathrm{C}$ profiles (as shown in Fig. 2a) for 4 weeks. The dark grey background indicates night-time. Error bars are s.e. m., $n=2-3$, average $>2.7$. c-f, VIN3 and various clock gene mRNA levels sampled on consecutive days at $8^{\circ} \mathrm{C}$ after 4 weeks constant $8^{\circ} \mathrm{C}$, $8 \mathrm{~h}$ photoperiod (purple). On the first day of sampling the plants were transferred to constant light conditions (blue). The lighter grey background indicates subjective night. RNA levels normalised to UBC, PP2A. $n=1-3$, average $>$ 2.1. $\mathbf{g}$ Summary of VIN3 regulation showing the slow regulator (B), the new component $(\boldsymbol{A})$ found to be necessary from modelling which gives the absence of warm regulation and the circadian clock, which could itself also be used as a temperature input 
reported slow upregulation in response to cold (Fig. 2b, Supplementary Figs. 5 and 6$)^{4,25-27}$. However, every night VIN3 expression returned to very low levels, again indicating that the absolute VIN3 mRNA level does not hold a memory of this slow cold exposure, as predicted by our previous analysis.

Given this diurnal regulation, we next returned to our model to re-evaluate our earlier conclusions. In fluctuating conditions, the daily maximum VIN3 levels increase with time under cold treatment (Supplementary Figs. 5 and 6) confirming the presence of the slow response. The degradation rate in the warm was estimated from plants that were transferred from the cold to the warm in the morning (Fig. 3c, d), when VIN3 levels would otherwise increase as part of its diurnal pattern (Fig. 4a). Therefore, the observed dynamics in Fig. $3 c$, $d$ will be a composite of the decrease due to the warm and the increase due to the diurnal regulation. Thus, the true degradation rate must be at least as fast as we observe. Hence, as with the model of Fig. 3a, such fast degradation would prevent the slow increase in VIN3 in fluctuating conditions. Therefore, we again conclude that VIN3 expression is regulated by at least two independent temperature inputs, a fast response to warmth and a slow response to cold, and also by a daily rhythm, that is either in addition to, or in combination with, one of the temperature inputs.

To understand whether the diurnal rhythm was a response to light or an influence of the circadian clock, we grew plants in short days and then transferred them to constant light and sampled during the transition. The VIN3 oscillation initially persisted at both mRNA and unspliced levels (Fig. 4c and Supplementary Fig. 7a), unlike for the clock oscillator genes $L U X$ ARRHYTHMO (LUX), PSEUDO-RESPONSE REGULATOR 5 (PRR5), PSEUDO-RESPONSE REGULATOR 9 (PRR9) and others (Fig. 4d, e, Supplementary Fig. 7b, c), which substantially lost cyclic expression within $16 \mathrm{~h}$. However, during the second day in constant light, the VIN3 oscillation was eventually lost, similar to the core oscillator CIRCADIAN CLOCK ASSOCIATED 1 (CCA1) (Fig. 4c, f and Supplementary Fig. 7a). These results are consistent with circadian regulation of transcription $^{30}$ and, more specifically, with the presence of 'Evening Element' binding sites in the proximal VIN3 promoter, to which CCA1 has been reported to bind ${ }^{31}$. The clock is also regulated in response to temperature ${ }^{32-34}$, suggesting that the circadian clock could form a further temperature input into VIN3 dynamics.

\section{Discussion}

These analyses have shown that, contrary to the current view, multiple and diverse aspects of the temperature profile are monitored to effect vernalisation. Transient cold temperatures (below around $14^{\circ} \mathrm{C}$, based on the vin3-4 mutant, Fig. 2d) during autumn, result in FLC transcriptional downregulation, but not epigenetic silencing. This is likely to involve cold-induced FLC antisense transcripts, whose expression is mutually exclusive with $F L C^{35}$. The subsequent epigenetic silencing of $F L C$, which is VIN3-dependent, has multiple temperature inputs that operate over different timescales. A schematic of these inputs is shown in Fig. 4g, although more work will be necessary to develop a full model of VIN3 that can be tested experimentally. However, from the existing data we can see that the combined action of these temperature inputs ensures that the major factor regulating VIN3 expression is the absence of spikes of warm temperature (above around $15^{\circ} \mathrm{C}$ ) each day. In the absence of these spikes (and therefore under continuous cold temperatures), daily peak VIN3 expression is permitted to slowly increase with accumulated time in the cold, in a degree days-like fashion. The exploitation of temperature extremes to generate a signal is reminiscent of a mechanism recently found in the process of seed germination ${ }^{36}$, where temperature fluctuations form a positive signal and induce seed germination. Variation sensitivity may therefore be a general and important mode of information processing in plants.

Separation of slow cold-memory and rapid response (Fig. 4g) also allows priming of VIN3 expression, where previous cold exposure results in faster initial upregulation in response to a second exposure, as observed in both Arabidopsis thaliana and Arabis alpina ${ }^{26,37}$. Previous modelling analyses have incorporated degree day-type dynamics ${ }^{9,11,14}$, which in Arabidopsis halleri have been shown to reflect an averaging of 6 weeks of previous temperature exposure ${ }^{10}$, but had not identified the requirement for absence of warm. A third input into VIN3 expression is through diurnal variations driven via the circadian clock, possibly via $\mathrm{CCA1}^{31}$. All three inputs (Fig. 4g) appear to regulate VIN3 principally through initiation of transcription. Although we do not know the molecular actors themselves, the slow nature of $\boldsymbol{B}$ is consistent with the previously observed accumulation of chromatin marks at $\operatorname{VIN}^{27}$. Furthermore, at least one component of the temperature response does not require new protein synthesis to allow VIN3 upregulation in cold temperatures ${ }^{38}$

Overall, we find that transient cool temperatures ('cold spikes', for example, the cool nights of autumn) are sufficient for the initial, temporary shutdown of FLC transcription. However, the absence of warm temperatures ('warm spikes') is the epigenetically recorded signature of winter for Arabidopsis thaliana (summarised in Supplementary Fig. 8). In the context of a warming and less stable climate, this sensitivity of developmental timing mechanisms to variability in temperature, rather than its average, could have large implications in ecological and agricultural contexts ${ }^{17,39-41}$.

\section{Methods}

Plant growth conditions and field experiments. The standard vernalisation reference accession $\mathrm{Col} F R I^{\mathrm{SF} 2}$ and the mutant vin3-4 FRI have been described previously 38,42 . For field experiments, seeds were stratified at $4{ }^{\circ} \mathrm{C}$ for 3 days and sown in $5.7 \mathrm{~cm}$ pot trays (Pöppelman, Lohne, Germany), using F2 compost (Levington Horticulture, Suffolk, UK) plus grit (Norwich) or P-soil (Hasselfors Örebro; Sweden), with six replicate cells per genotype per timepoint for RNA samples. For flowering time assays, seeds were sown in $3.9 \mathrm{~cm}$ pot trays (Pöppelman) and thinned to a single plant per cell after germination, with 36 plants per genotype.

In Norwich, trays were placed on benches in a well-ventilated, unheated, unlit glasshouse, and bedded in vermiculite to simulate bedding in soil. Trays were covered with plastic lids or cling film for the first week to ensure germination, and watered when necessary. Plants were sown on 29 September 2014. Seedlings were thinned as necessary until only three per pot were left. Genotypes were randomised $^{43}$ within six single-replicate sample-sets per timepoint, and sample-sets were then randomised in a three-block design lengthwise along the greenhouse, with some adjustment to ensure each of the two replicates per block were on different benches. TinyTag Plus 2 dataloggers (Gemini Data Loggers (UK) Ltd) were placed on all benches and in each block at plant level. The lower temperature limit observed of $\sim 2{ }^{\circ} \mathrm{C}$ probably resulted from buffering of the lowest temperatures by glasshouse confinement, as loggers placed just outside the glasshouse or very near the vents mirrored internal temperatures closely except below $\sim 4{ }^{\circ} \mathrm{C}$.

For sampling, aerial parts of seedlings (up to 5 weeks) and thereafter the young leaves and meristems were taken from at least three plants per sample, excluding flowering bolts where applicable, and flash frozen in liquid nitrogen. Sampling was performed between 1145-1300 hours.

In Sweden, trays were initially grown outside under plastic covers for 2 weeks at Mid Sweden University, Sundsvall (Sweden North) or Lund University (Sweden South) to ensure germination. Trays were then moved to the experiment sites (North: Ramsta $\left(62^{\circ} 50.988^{\prime} \mathrm{N}, 18^{\circ} 11.570^{\prime} \mathrm{E}\right)$, South: Ullstorp $\left(56^{\circ} 06.6721^{\prime} \mathrm{N}, 13^{\circ}\right.$ $\left.94.4655^{\prime} \mathrm{E}\right)$ ) and the trays dug into the soil. Plants were sown and moved on: North Set first planting sown 26 August 2014, moved 11 September; North Set second planting sown 8 September 2014, moved 24 September; South sown 24 September 2014, moved 8 October. Timepoint sample-sets were randomised in three blocks. Trays were watered when necessary. TinyTag Plus 2 dataloggers were placed with the plants at soil level.

For sampling, whole plants were rinsed, dried on paper, placed in sample tubes and frozen on dry ice. Sampling times were between 1100 and 1700 hours and precise times were recorded on each occasion. 
Flowering time was scored as the first day of the floral transition being visible at the apical meristem.

Laboratory experiments. Plants were sown as for the Norwich glasshouse, but on P24 tray cells (Desch PlantPak, Waalwijk, Netherlands) covered with fine net curtain to allow clean extraction of seedlings. Plants were initially grown at $22^{\circ} \mathrm{C}$ $16 \mathrm{~h}$ day $/ 20^{\circ} \mathrm{C} 8 \mathrm{~h}$ night for one week ('NV', Non Vernalised), before moving to Panasonic MLR-352 series growth cabinets set to 37-52 $\mu \mathrm{mol}$ light (setting 3 ) for 8 $\mathrm{h}$ per day and the described temperature setting. For the $24 \mathrm{~h}$ experiment in Supplementary Fig. 5, an alternative 'NV' temperature regime was used in the Panasonic cabinets as described in the figure. For Fig. 2e, and Supplementary Fig. 3b plants were returned to 'NV' conditions after vernalisation treatment in the cabinets. For Supplementary Fig. 3b, flowering time plants were also pricked out into individual P24 cells after transfer. For the post-cold degradation experiments of Fig. $3 \mathrm{c}$, d, following the standard NV treatment, plants were moved to $5^{\circ} \mathrm{C} 8 \mathrm{~h}$ photoperiod walk-in vernalisation room for 4 weeks and finally transferred to a growth cabinet set to $22^{\circ} \mathrm{C} 16 \mathrm{~h}$ photoperiod during sampling. For Fig. $4 \mathrm{c}-\mathrm{f}$ and Supplementary Fig. 7 plants were grown on Murashige and Skoog (MS) agar plates without glucose. They were grown at $20^{\circ} \mathrm{C} 8 \mathrm{~h}$ photoperiod in a growth cabinet for 4 weeks and then moved to $8{ }^{\circ} \mathrm{C} 8 \mathrm{~h}$ photoperiod for 26 days before sampling. On the 26 th night, they were separated so that the ' $8{ }^{\circ} \mathrm{C}$ Light' samples were treated with constant light.

Sample sizes and replication. For field experiments, six replicates per genotype were chosen to allow for losses in the field and for duplication within randomisation blocks. For flowering time, within space constraints enough individual plants were sown to ensure at least ten samples per randomisation block/condition. For cabinet experiments, three biological or technical replicates were sampled per timepoint to allow sufficient number of samples for statistical analysis while allowing for space constraints within cabinets. Separate sowing dates were used for three replicates of experiments for Figs. 2b-d, 3b, e and 4a, b and Supplementary Figs. 5 and 6, with multiple timepoints sampled. For Fig. 2e, separate sowing dates were used for two replicates of the experiment and two technical replicates were sampled within each experiment. For Figs. $3 c$, d and $4 c-f$, and Supplementary Figs. 7 and 9 three technical replicates were taken from the same experiment. Where resulting samples are smaller, this is due to experimental or processing loss (e.g., death of plants in the field, or degradation due to poor sample quality or processing, see 'RNA preparation and QPCR'). Final sample sizes for all data points can be found in Supplementary Data 1.

RNA preparation and QPCR. RNA was extracted as described ${ }^{44}$, using phenol equilibrated to $\mathrm{pH}$, followed by lithium chloride precipitation. RNA was DNase treated with Turbo DNA Free DNase, lithium precipitated, then treated with SuperScript Reverse Transcriptase III (both Life Technologies) and gene-specific primers (VIN3 qPCR 1R, FLC spliced R, UBC_qPCR_R, PP2A R2, FLC unspliced_RP 5'-CTTTGTAATCAAAGGTGGAGAGC-3', VIN3 unspliced R, in one reaction, UBC qPCR_R, PP2A R2, LUX R, GI R, JF119-CCA1-R, JF117-TOC1-R, JF310-PRR9-R, JF283-PRR5-R in another) to synthesise cDNA. QPCR was performed using SYBRGreen Master Mix II on a LightCycler 480 II (both Roche) using primer pairs: VIN3 $\mathrm{qPCR}$ 1F $5^{\prime}$-TGCTTGTGGATCGTCTTGTCA-3' and VIN3 qPCR 1R 5'-TTCTCCAGCATCCGAGCAAG-3', FLC spliced F 5'-AGCCAAGAAGACCGAACTCA- $3^{\prime}$ and FLC spliced R $5^{\prime}$-TTTGTCCAGCAGGTGACATC-3', VIN3 unspliced F 5'-GGTTTTATTGCGCGTATTGC-3' and VIN3 unspliced R $5^{\prime}$-CCAGCATCTGTAAGCACTCG-3', UBC qPCR F 5'CTGCGACTCAGGGAATCTTCTAA- $3^{\prime}$ and UBC qPCR R $5^{\prime}$-TTGTGCCATTGAATTGAACCC-3', PP2A F2 5'-ACTGCATCTAAAGACAGAGTTCC-3' and PP2A R2 5'-CCAAGCATGGCCGTATCATGT-3', LUX F 5'-AGGTGGAAGCGCAAATGAGA- $3^{\prime}$ and LUX R $5^{\prime}$-TGTAGACACCAAGAACCTATCTCT- ${ }^{\prime}$, and GI F $5^{\prime}$-GTATCTGCAACGCCAGCGA-3' and GI R $5^{\prime}$-GCAACTCCCTTTCAGCCTGA- $3^{\prime 30}$, JF118-CCA1-F $5^{\prime}$-CTGTGTCTGACGAGGGTCGAA- $3^{\prime}$ and JF119-CCA1-R 5' -ATATGTAAAACTTTGCGGCAATACCT-3', JF116-TOC1-F 5'-ATCTTCGCAGAATCCCTGTGATA-3' and JF117-TOC1-R 5'-GCACCTAGCTTCAAGCACTTTACA-3', JF309-PRR9-F 5'-GATTGGTGGAATTGACAAGC- $3^{\prime}$ and JF310-PRR9-R 5'-TCCTCAAATCTTGAGAAGGC- $3^{\prime}$, JF282PRR5-F $5^{\prime}$-GTGTATGTTGAAAGGTGCGG-3' and JF283-PRR5-R 5'-AGGAGCAAGTGAAGTTTGTC- $3^{\prime 45}$

Test amplicons were normalised to the geometric mean of two standard reference genes, At1g13320 ('PP2A') and At5g25760 ('UBC' ${ }^{46,47}$. Samples with very low values of control genes (indicating degradation, inhibition or sample loss) and data for amplicons that were below the detection limit were excluded. The final sample size for all experiments can be found in Supplementary Data 1. For the field experiments and for the experiment shown in Supplementary Fig. 5, the output was analysed using LinReg ${ }^{48}$ and in order to normalise across a large number of samples, samples were further normalised to a Control Sample run on every QPCR plate. The Control cDNA was made in the same way as the test samples from an equal mix of RNA from 8 weeks cold-treated $\mathrm{Col} F I^{\mathrm{S}}$ 2 and from non-vernalised Col $F R I^{\mathrm{SF} 2}$ to ensure expression of all amplicons and avoid divide-by-zero error, mixed, prediluted and aliquoted to ensure consistency. For other expression experiments, smaller sample numbers allowed simultaneous processing, so $\mathrm{Ct}$ values from the LightCycler software were analysed using the $\Delta \Delta \mathrm{Ct}$ method.
Mathematical model. The model was solved numerically ${ }^{49}$ with initial conditions (indicated by the subscript 0): $v_{0}=0$ and $r_{0}=0$ for Fig. $3 e$ and $v_{0}=0.124$ and $r_{0}=$ $d_{1} v_{0} / p_{1}$ for Fig. $3 \mathrm{~d}$. Due to the simplicity of the model, parameters could be fitted to the data of Fig. 3d, e (constant temperature only) by least squares fitting for $p_{1}$ and manual adjustment for other parameters: $p_{1}=0.91$ day $^{-1}, d_{1}=100$ day $^{-1}, d_{2}=7$ day ${ }^{-1}$. Fig $3 \mathrm{~d}$ provides lower bounds for $d_{1}$ and $d_{2}$. The conclusion of the model is not sensitive to the parameters except the decay rate of $\boldsymbol{r}$ in the warm $\left(d_{2}\right)$ which is constrained by the data. The temperature dependence of $d_{r}(T)$ is chosen as a step function at $22^{\circ} \mathrm{C}$ so as to use the simplest and most conservative form. Using a temperature step higher than at $22^{\circ} \mathrm{C}$ would not replicate the fast degradation observed in Fig. 3c, d, while a step at a lower temperature would lead to similar results in 14-fluctuating conditions but with even lower VIN3 levels. A more complex temperature dependence for the production/degradation of $\boldsymbol{r}$ is possible and indeed necessary to capture the full degree days-like accumulation of VIN3 at different constant cold temperatures. However, even with such additional complexity, the fluctuating temperature behaviour of VIN3 is not captured and therefore the conclusion of the need for two independent thermosensory pathways is not affected. An additional model considering unspliced VIN3 as an intermediate was also tested and the conclusions of the model were the same (Supplementary Fig. 9)

Statistics. One-tailed $t$-test was performed to test for reactivation after the cold (Fig. 2e) $\left(\mathrm{H}_{0}\right.$ : The true mean difference $F L C_{\mathrm{T} 7}-F L C_{\mathrm{T} 0}$ is less than or equal to 0$)$. Samples from the same experimental replicate that had experienced the same length of vernalisation treatment were paired. Samples here refer to the mean of any technical replicates within the experimental replicate. The same test was performed for the 14-constant treatment (not significant, $p=0.7480$ ) and the 14fluctuating treatment (significant, $p=0.0064$ ), using the Matlab function ttest $($ ).

To compare the rate of decrease of FLC in the field (Fig. 1 and Results section ' $F L C$ silencing can be separated into two phases in the field'), ANCOVA was performed using the R function $\operatorname{lm}()^{50}$.

Code availability. The authors declare that all code supporting the findings of this study is available from the corresponding author upon request.

Data availability. The authors declare that all data supporting the findings of this study are included in the main manuscript file or Supplementary Information or are available from the corresponding author upon request.

Received: 27 October 2017 Accepted: 17 January 2018 Published online: 12 February 2018

\section{References}

1. Sheldon, C. C. et al. Resetting of FLOWERING LOCUS C expression after epigenetic repression by vernalization. Proc. Natl. Acad. Sci. USA 105, 2214-2219 (2008).

2. Gendall, A. R., Levy, Y. Y., Wilson, A. \& Dean, C. The VERNALIZATION 2 gene mediates the epigenetic regulation of vernalization in Arabidopsis. Cell 107, 525-535 (2001).

3. Bastow, R. et al. Vernalization requires epigenetic silencing of FLC by histone methylation. Nature 427, 164-167 (2004)

4. Sung, S. \& Amasino, R. M. Vernalization in Arabidopsis thaliana is mediated by the PHD finger protein VIN3. Nature 427, 159-164 (2004).

5. De Lucia, F., Crevillen, P., Jones, A. M. E., Greb, T. \& Dean, C. A PHD polycomb repressive complex 2 triggers the epigenetic silencing of FLC during vernalization. Proc. Natl. Acad. Sci. USA 105, 16831-16836 (2008).

6. Napp-Zinn, K. Untersuchungen über das Vernalisationsverhalten einer winterannuellen Rasse von Arabidopsis thaliana. Planta 50, 177-210 (1957).

7. Wollenberg, A. C. \& Amasino, R. M. Natural variation in the temperature range permissive for vernalization in accessions of Arabidopsis thaliana. Plant Cell Environ. 35, 2181-2191 (2012).

8. Duncan, S. et al. Seasonal shift in timing of vernalization as an adation to extreme winter. eLife 4, e06620 (2015).

9. Wilczek, A. M. et al. Effects of genetic perturbation on seasonal life history plasticity. Science 323, 930-934 (2009).

10. Aikawa, S., Kobayashi, M. J., Satake, A., Shimizu, K. K. \& Kudoh, H. Robust control of the seasonal expression of the Arabidopsis FLC gene in a fluctuating environment. Proc. Natl. Acad. Sci. USA 107, 11632-11637 (2010).

11. Satake, A. et al. Forecasting flowering phenology under climate warming by modelling the regulatory dynamics of flowering-time genes. Nat. Commun. 4 2303 (2013)

12. Kudoh, H. Molecular phenology in plants: in natura systems biology for the comprehensive understanding of seasonal responses under natural environments. New Phytol. 210, 399-412 (2016)

13. Bond, D. M., Dennis, E. S. \& Finnegan, E. J. The low temperature response pathways for cold acclimation and vernalization are independent. Plant Cell Environ. 34, 1737-1748 (2011). 
14. Chew, Y. H. et al. An augmented Arabidopsis phenology model reveals seasonal temperature control of flowering time. New Phytol. 194, 654-665 (2012).

15. Wurr, D. C. E., Fellows, J. R. \& Phelps, K. Crop scheduling and prediction Principles and opportunities with field vegetables. Adv. Agron. 76, 201-234 (2002).

16. Wang, E. \& Engel, T. Simulation of phenological development of wheat crops. Agric. Syst. 58, 1-24 (1998).

17. Luedeling, E., Girvetz, E. H., Semenov, M. A. \& Brown, P. H. Climate change affects winter chill for temperate fruit and nut trees. PLoS ONE 6, e20155 (2011).

18. Buzas, D. M., Robertson, M., Finnegan, E. J. \& Helliwell, C. A. Transcriptiondependence of histone $\mathrm{H} 3$ lysine 27 trimethylation at the Arabidopsis polycomb target gene FLC. Plant J. Cell Mol. Biol. 65, 872-881 (2011).

19. Hosogane, M., Funayama, R., Nishida, Y., Nagashima, T. \& Nakayama, K. Ras-induced changes in $\mathrm{H} 3 \mathrm{~K} 27 \mathrm{me} 3$ occur after those in transcriptional activity. PLoS Genet. 9, el003698 (2013).

20. Riising, E. M. et al. Gene silencing triggers polycomb repressive complex 2 recruitment to CpG islands genome wide. Mol. Cell 55, 347-360 (2014).

21. Swiezewski, S., Liu, F., Magusin, A. \& Dean, C. Cold-induced silencing by long antisense transcripts of an Arabidopsis Polycomb target. Nature 462, 799-802 (2009).

22. Helliwell, C. A., Robertson, M., Finnegan, E. J., Buzas, D. M. \& Dennis, E. S. Vernalization-repression of Arabidopsis FLC requires promoter sequences but not antisense transcripts. PLoS ONE 6, e21513 (2011).

23. Burghardt, L. T. et al. Fluctuating, warm temperatures decrease the effect of a key floral repressor on flowering time in Arabidopsis thaliana. New Phytol. 210, 564-576 (2016).

24. Shindo, C., Lister, C., Crevillen, P., Nordborg, M. \& Dean, C. Variation in the epigenetic silencing of FLC contributes to natural variation in Arabidopsis vernalization response. Genes Dev. 20, 3079-3083 (2006).

25. Greb, T. et al. The PHD finger protein VRN5 functions in the epigenetic silencing of Arabidopsis FLC. Curr. Biol.17, 73-78 (2007).

26. Bond, D. M., Dennis, E. S., Pogson, B. J. \& Finnegan, E. J. Histone acetylation, VERNALIZATION INSENSITIVE 3, FLOWERING LOCUS C, and the vernalization response. Mol. Plant 2, 724-737 (2009).

27. Finnegan, E. J. et al. Polycomb proteins regulate the quantitative induction of VERNALIZATION INSENSITIVE 3 in response to low temperatures. Plant J. Cell Mol. Biol. 65, 382-391 (2011).

28. Kim, D.-H., Zografos, B. R. \& Sung, S. Vernalization-mediated VIN3 induction overcomes the LIKE-HETEROCHROMATIN PROTEIN1/ POLYCOMB REPRESSION COMPLEX2-mediated epigenetic repression. Plant Physiol. 154, 949-957 (2010).

29. Yang, H. et al. Distinct phases of Polycomb silencing to hold epigenetic memory of cold in Arabidopsis. Science 357, 1142-1145 (2017)

30. Bieniawska, Z. et al. Disruption of the Arabidopsis circadian clock is responsible for extensive variation in the cold-responsive transcriptome. Plant Physiol. 147, 263-279 (2008).

31. Nagel, D. H. et al. Genome-wide identification of CCA1 targets uncovers an expanded clock network in Arabidopsis. Proc. Natl. Acad. Sci. USA 112, E4802-E4810 (2015).

32. Sanchez, S. E. \& Kay, S. A. The plant circadian clock: from a simple timekeeper to a complex developmental manager. Cold Spring Harb. Perspect. Biol. 8, a027748 (2016).

33. Salomé, P. A., Weigel, D. \& McClung, C. R. The role of the Arabidopsis morning loop components CCA1, LHY, PRR7, and PRR9 in temperature compensation. Plant Cell 22, 3650-3661 (2010).

34. Gould, P. D. et al. The molecular basis of temperature compensation in the Arabidopsis circadian clock. Plant Cell Online 18, 1177-1187 (2006).

35. Rosa, S., Duncan, S. \& Dean, C. Mutually exclusive sense-antisense transcription at FLC facilitates environmentally induced gene repression. Nat. Commun. 7, 13031 (2016).

36. Topham, A. T. et al. Temperature variability is integrated by a spatially embedded decision-making center to break dormancy in Arabidopsisseeds. Proc. Natl. Acad. Sci. USA 114, 6629-6634 (2017).

37. Castaings, L. et al. Evolutionary conservation of cold-induced antisense RNAs of FLOWERING LOCUS C in Arabidopsis thaliana perennial relatives. Nat. Commun. 5, 4457 (2014).

38. Bond, D. M., Wilson, I. W., Dennis, E. S., Pogson, B. J. \& Jean Finnegan, E. VERNALIZATION INSENSITIVE 3 (VIN3) is required for the response of Arabidopsis thaliana seedlings exposed to low oxygen conditions. Plant. J. 59, 576-587 (2009).

39. Atkinson, C. J., Brennan, R. M. \& Jones, H. G. Declining chilling and its impact on temperate perennial crops. Environ. Exp. Bot. 91, 48-62 (2013).

40. Laube, J. et al. Chilling outweighs photoperiod in preventing precocious spring development. Glob. Change Biol. 20, 170-182 (2014).

41. Wang, E. et al. The uncertainty of crop yield projections is reduced by improved temperature response functions. Nat. Plants 3, 17102 (2017)
42. Lee, I. \& Amasino, R. M. Effect of vernalization, photoperiod, and light quality on the flowering phenotype of Arabidopsis plants containing the FRIGIDA gene. Plant Physiol. 108, 157-162 (1995).

43. Urbaniak, G. C. \& Plous, S. Research Randomizer. Version 4.0 (2015).

44. Box, M. S., Coustham, V., Dean, C. \& Mylne, J. S. Protocol: a simple phenol based method for 96-well extraction of high quality RNA from Arabidopsis. Plant Methods 7, 7 (2011).

45. MacGregor, D. R. et al. HIGH EXPRESSION OF OSMOTICALLY RESPONSIVE GENES1 is required for circadian periodicity through the promotion of nucleo-cytoplasmic mRNA export in Arabidopsis. Plant Cell. 25 4391-4404 (2013)

46. Czechowski, T., Stitt, M., Altmann, T., Udvardi, M. K. \& Scheible, W.-R. Genome-wide identification and testing of superior reference genes for transcript normalization in Arabidopsis. Plant Physiol. 139, 5-17 (2005).

47. Yang, H., Howard, M. \& Dean, C. Antagonistic roles for H3K36me3 and H3K27me3 in the cold-induced epigenetic switch at Arabidopsis FLC. Curr. Biol. 24, 1793-1797 (2014).

48. Ruijter, J. M. et al. Amplification efficiency: linking baseline and bias in the analysis of quantitative PCR data. Nucleic Acids Res. 37, e45-e45 (2009).

49. Shampine, L. \& Reichelt, M. The MATLAB ODE Suite. SIAM J. Sci. Comput 18, 1-22 (1997)

50. R. Core Team. R: A Language and Environment for Statistical Computing. (R Foundation for Statistical Computing: Vienna, Austria, 2016).

\section{Acknowledgements}

The authors would like to thank the entire Dean group and friends for help with the field experiments; Huamei Wang and Annie McGee for seed preparation; family Öhman (Ramsta) and Nils Jönsson (Ullstorp) for hosting the field experiments and experimentalists in Sweden; Catherine Taylor and JIC Horticultural Services for plant care in Norwich; Dr. Jie Song (Imperial College London) for the VIN3 unspliced primers; the Dean group, Dr. Giuseppe Facchetti, Dr. Cecilia Lövkvist, Dr. Laura Dixon, Dr. Xiaoqi Feng and Dr. Steven Penfield (John Innes Centre), Dr. Melissa Antoniou-Kourounioti (University of East Anglia), Dr. Akiko Satake (Kyushu University) and Dr. Karissa Sanbonmatsu (Los Alamos National Laboratories) for discussion; and Ingalill Thorsell of Drakamöllan Gårdshotell for inspirational cooking. This work was funded by the European Research Council grant 'MEXTIM', and supported by the BBSRC Institute Strategic Programmes GRO (BB/J004588/1) and GEN (BB/P013511/1).

\section{Author contributions}

J.H. and R.L.A.-K. designed experiments, acquired data and analysed data for all figures. R.L.A.-K. and M.H. performed mathematical modelling. C.S., K.B., S.H. and T.S. designed experiments, acquired data and analysed data, J.I. designed experiments, D.C. and B.R.C.H. designed experiments and acquired data for Fig. 1. R.H.B. designed experiments, acquired data and analysed data for Figs. 2-4. C.D. and M.H. are responsible for the concept of the research, designed experiments and analysed data. R.L. A.-K., J.H., M.H. and C.D. wrote the paper.

\section{Additional information}

Supplementary Information accompanies this paper at https://doi.org/10.1038/s41467018-03065-7.

Competing interests: The authors declare no competing financial interests.

Reprints and permission information is available online at http://npg.nature.com/ reprintsandpermissions/

Publisher's note: Springer Nature remains neutral with regard to jurisdictional claims in published maps and institutional affiliations.

\footnotetext{
Attribution 4.0 International License, which permits use, sharing, adaptation, distribution and reproduction in any medium or format, as long as you give appropriate credit to the original author(s) and the source, provide a link to the Creative Commons license, and indicate if changes were made. The images or other third party material in this article are included in the article's Creative Commons license, unless indicated otherwise in a credit line to the material. If material is not included in the article's Creative Commons license and your intended use is not permitted by statutory regulation or exceeds the permitted use, you will need to obtain permission directly from the copyright holder. To view a copy of this license, visit http://creativecommons.org/ licenses/by/4.0/.
}

Open Access This article is licensed under a Creative Commons

(c) The Author(s) 2018 